\title{
Percepciones Docentes en torno a la Presencia de Niños y Niñas Migrantes en Escuelas de Santiago: Retos y Desafíos para la Inclusión
}

\section{Teachers' Perceptions Regarding the Presence of Migrant Boys and Girls in Schools of Santiago: Challenges for Inclusion}

\author{
Leidy Cerón ${ }^{1 *}$ \\ Marly Pérez Alvarado ${ }^{2}$ \\ Rolando Poblete ${ }^{3}$ \\ ${ }^{1}$ Grupo de gestión de la sociedad educacional Carlos Salinas \\ ${ }^{2}$ Corporación Educacional Laudelina Araneda \\ ${ }^{3}$ Universidad Central de Chile
}

La llegada de población migrante en edad escolar a Chile ha generado un conjunto de desafíos para el Estado, el sistema educativo y en especial para las escuelas que reciben a niños y niñas extranjeras, instalando la preocupación por alcanzar la inclusión y el respeto de los derechos de todos y todas. Al respecto, son múltiples los factores que intervienen en este proceso, sin embargo, la posibilidad de avanzar hacia la inclusión educativa en escuelas con presencia de niños y niñas de orígenes diversos, está mediada en buena medida por la valoración que tengan los docentes acerca de sus estudiantes. Bajo tales premisas, la investigación que da origen a este artículo se propuso conocer la percepción que tienen los y las profesoras de dos escuelas de la comuna de Santiago respecto de la inclusión educativa de estudiantes migrantes en sus escuelas, y las posibilidades que se vislumbran para la construcción de una cultura inclusiva. A través de una metodología mixta, que combinó la aplicación de encuestas y entrevistas semiestructuradas se pudo establecer que los y las docentes poseen una visión restringida de la inclusión, asumiendo que los migrantes presentan una serie de déficits académicos y de comportamiento que dificultan su proceso de inclusión y su plena participación en las escuelas.

Descriptores: Migración, Cultura, Educación, Profesor, Percepción.

The arrival of a migrant population of school age in Chile has created a set of challenges for the State, the education system and especially for schools that receive foreign children, establishing a concern to achieve inclusion and respect for rights of all. In this regard, there are many factors that intervene in this process, however, the possibility of advancing towards inclusive education in schools with boys and girls of diverse origins is largely mediated by the appraisal teachers have of their students. Under this premise, the aim of the research that originated this article was to get to know the perception teachers have in two schools of the borough of Santiago, about inclusive education of the migrant students in their schools, and the possibilities for the construction of a culture of inclusion. Through a mixed methodology with surveys and semi structured interviews, it was possible to establish that these teachers have a restricted view of inclusion, and that they assume that migrants have a series of academic and behavioral shortcomings that hinder their inclusion and full participation in schools.

Keywords: Migration, Culture, Education, Teacher, Perception.

*Contacto: leydiceron@gmail.com

ISSN: 0718-7378

www.rinace.net/rlei/
Recibido: 30/06/2017



Aceptado: 26/09/2017 


\section{Antecedentes}

La presencia de población migrante en las aulas habitualmente plantea desafíos al sistema educacional en todos sus niveles, abriendo un debate que implica posiciones político pedagógicas, expectativas educativas y también sociales (Jiménez y Fardella, 2015). Muchas de las discusiones que se generan en ese marco remiten a la posibilidad de alcanzar una cultura inclusiva al interior de las escuelas, casi como un mandato propio de las sociedades actuales que ubican el respeto de la diversidad en el sistema educativo como un derecho de las personas (UNESCO, 2001), demandando también la formación de ciudadanos multiculturales capaces de desenvolverse en un mundo global (Johnson, 2015). En tal sentido la inclusión, y en especial la construcción de una cultural inclusiva, se sostienen en una perspectiva basada en los derechos humanos en la cual todos y todas acceden a la educación al margen de cualquier consideración relacionada con la etnia, género, estilos de aprendizaje o necesidades educativas especiales (UNESCO, 2005).

Pese a lo anterior, la rápida expansión de la inmigración y su magnificación por los medios de comunicación han hecho aflorar posturas menos amables hacia los/as extranjeros (León, Mira y Gómez, 2007), incluidas acciones abiertamente contrarias a ellos/as, como ha ocurrido en Chile a propósito de la marcha llevada a cabo en Antofagasta el año 2013 en contra de los inmigrantes colombianos, que dio origen a una polémica nacional sobre su presencia. Muchas de esas actitudes también se replican en las escuelas (Pavez, 2012; Poblete y Galaz, 2015; Tijoux, 2013; UNICEF, 2004, 2011), de forma tal que su experiencia no siempre se condice con los principios de la inclusión. De hecho, la evidencia indica que la escuela chilena se ordena desde una perspectiva normalizadora y excluyente apostando por la construcción de una identidad uniforme y homogénea (Poblete y Galaz, 2007).

Como muestran los datos existentes a nivel nacional, la matrícula extranjera en las escuelas del país se ha incrementado en el último tiempo alcanzando a cerca de 77.000 niños y niñas el año 2017, de los cuales el 41,3\% se encuentra en situación irregular (Ministerio de Educación de Chile, 2017). Aunque representan cerca del 2\% de la matrícula total del sistema, su presencia y mayor visibilidad ha desafiado a las escuelas a construir una cultura inclusiva en que todos y todas sean respetados.

Pese a la existencia de instrumentos legales que aseguran el acceso y progreso de los/as estudiantes migrantes en el sistema educacional (Ministerio de Educación de Chile 2005, 2016), es claro que estos no garantizan por sí mismos el respeto de los derechos consagrados en las normas o decretos, ni tampoco la inclusión -más allá del acceso- en las escuelas a las que asisten. Un punto a tener en cuenta al respecto es el rol de los/as docentes y la percepción o valoración que pueden tener acerca de la presencia de niños y niñas migrantes en sus escuelas y aulas, toda vez que si esta es positiva son mayores las posibilidades de avanzar hacia la instalación de políticas y culturas inclusivas (León, Mira y Gómez, 2007).

Bajo tales premisas la investigación que da origen a este artículo se planteó como objetivo conocer la percepción que tienen los y las docentes en dos escuelas de la comuna de Santiago respecto de la inclusión educativa de estudiantes migrantes en sus aulas y las posibilidades que se vislumbran desde ahí para la construcción de una cultura inclusiva en tales centros. 


\section{Alcances teóricos}

La inclusión educativa remite a valores y prácticas fundados en los derechos humanos (Blanco, 1999; Booth y Ainscow, 2000; Echeita, 2008), dentro de los cuales no tiene cabida la discriminación o cualquier situación que menoscabe la dignidad de las personas; en esos términos plantea analizar las políticas, las prácticas y las culturas para eliminar las barreras que impiden el acceso y participación de todos en el sistema educativo, lo que por cierto implica reestructurar esos mismos elementos para que los centros educativos puedan atender a la diversidad del alumnado (Booth y Ainscow, 2000).

Aunque esto opera muchas veces como un ideal, es evidente que también está mediado por las actitudes y percepciones que los integrantes de una comunidad educativa tienen acerca de ellos mismos. En tal sentido, las instituciones educativas que albergan a estudiantes pertenecientes a grupos culturales diversos ofrecen numerosos hechos de interés en su interior (Carrasco, 1998; Ogbu, 1991; Poblete y Galaz, 2007), situando muchas veces en los/as docentes una responsabilidad significativa en cuanto al tratamiento y abordaje de la diversidad. Al respecto, Jordán (1994) sostiene que lo más relevante -incluso más que las competencias que pueden desplegar los y las docentes- son las actitudes que tienen hacia la diversidad. En esa línea, Ortiz (2008) señala que el incremento del alumnado extranjero en las aulas está siendo percibido como una amenaza y como uno de los principales problemas del sistema educativo, y que el discurso expresado por docentes se sitúa desde una posición asimilacionista en la que las diferencias de cualquier orden deben ser suprimidas. Esas “ideologías prejuiciosas son las que finalmente inciden en el contexto escolar, en las relaciones y los procesos de enseñanza-aprendizaje que tienen lugar en él” (Ortiz, 2008, p. 254).

El problema radicaría en que niños y niñas inmigrantes presentan mayores dificultades para recibir un proceso de enseñanza efectivo y adaptarse a los requerimientos de disciplina, orden, conocimiento del lenguaje y los códigos del sistema escolar (Kaluf, 2009). Aunque en ciertos casos los y las docentes tienen actitudes favorables hacia la presencia de estudiantes extranjeros, persiste -la mayor parte de las veces- la percepción que alteran el funcionamiento de las clases, a la vez de un sentimiento de compasión que podría afectar negativamente su rendimiento académico y su integración (Castro y Álvarez, 2005).

Respecto de la forma de abordar la diversidad al interior de las aulas, Kaluf (2009) afirma que la mayoría de los docentes se encuentran poco preparados y tienden a considerar cualquier tipo de diferenciación como una forma de discriminación, por lo que trabajan bajo la ilusión del trato igualitario, asumiendo que, porque no existe la diferencia, no hay discriminación. Esto es corroborado también por Castro (2012), al señalar que los docentes asumen que niños y niñas migrantes no requieren de apoyos o tratos diferenciados ya que son considerados como iguales a los estudiantes nacionales, por tanto, no modifican sus prácticas, lo que incide en parte en la invisibilización de la diversidad presente en el aula.

Otros/as docentes perciben la diversidad cultural en sus salas con mayor sensibilidad, pero no reconocen que esto implica cambios en sus prácticas, por lo cual no generan un impacto positivo en los procesos de inclusión. Finalmente, hay otros dispuestos a indagar y conocer sobre los principios de la interculturalidad, respetando las diferencias y 
considerando los aspectos que entran en juego en centros educativos que cuentan con presencia de niños inmigrantes (Salas et al., 2012).

A medida que los y las docentes se sienten más confiados en su labor, tienden a generar expectativas positivas, lo que depende en buena medida del grado de interés que hayan tenido en comprender las diferencias y no guiarse exclusivamente por prejuicios (Cerrutti y Binstock, 2012).

Es claro que la importancia de las percepciones que tengan los docentes respecto de la migración está en el hecho que de estas dependerá no solo el trato que otorguen a los/as estudiantes o su rendimiento (Jordán, 1994; León, Mira y Gómez, 2007), sino también la posibilidad de aportar a una cultural inclusiva al interior de las escuelas, entendida como una comunidad que promueve valores coherentes con los principios de la democracia, solidaridad y el respeto irrestricto a la diversidad (Blanco, 1999, 2005; Booth y Aisncow, 2000; Echeita, 2008).

Ahora bien, la idea de cultura inclusiva remite a dos elementos, por un lado, a la construcción de una comunidad y, por otro, a la presencia de valores inclusivos en las escuelas (Booth y Aisncow, 2000). Sin embargo, lo que debe prevalecer es la modificación de los prejuicios y estereotipos con los que docentes y padres de familia han crecido para construir un "clima escolar favorable en donde los docentes sean el fiel reflejo de la práctica de valores como el respeto, la colaboración, la justicia, la identidad” (Lasso, 2015, p. 24). En términos más concretos, Dyson, Howes y Roberts (2002), luego de una revisión exhaustiva de la literatura en torno a los procesos de inclusión concluyen que los rasgos comunes a las culturas inclusivas son el acuerdo entre los adultos referente a los valores del respeto por la diferencia y compromiso con todos los estudiantes para que tengan acceso a las oportunidades de aprendizaje; colaboración entre el profesorado para la resolución de problemas; presencia de líderes comprometidos con los valores inclusivos y con los cambios de las estructuras tradicionales, de forma tal de avanzar hacia otras más integradoras.

\section{Metodología}

El estudio realizado tuvo un carácter mixto, vale decir, se aplicaron técnicas cuantitativas y cualitativas para el proceso de recolección de información, considerando lo señalado por Pereira (2012) en relación a que los diseños mixtos permiten la obtención de una mejor evidencia y comprensión de los fenómenos de estudio. Dos fueron las variables sobre las cuales se llevó a cabo la indagación, por un lado, construcción de comunidad, subdividida en dos categorías de análisis: participación de niños y niñas migrantes en las actividades de la escuela y relaciones interpersonales; y, por otro, valores inclusivos que se generan por la presencia de niños y niñas migrantes, que a su vez se operacionalizó en respeto y valoración de la diversidad e igualdad de oportunidades educativas. Para ambos casos se buscó conocer la percepción y valoración de los/as docentes.

La investigación se realizó en dos escuelas básicas de la comuna de Santiago cuya matrícula de niños/as extranjeros/as fluctúa entre un $45 \%$ y $50 \%$ del total de estudiantes. En cada establecimiento se aplicó un cuestionario tipo Likert a una muestra de 37 docentes, que representa el $70 \%$ del total de profesores/as para ambos casos de estudio. El cuestionario fue previamente validado a través de un pre test aplicado a docentes en una escuela de similares características, asegurando la validez de constructo requerida 
para este tipo de instrumentos (Hernández, Fernández y Baptista, 2010). Para el análisis de los datos se construyeron intervalos de puntuación para identificar la presencia de percepciones y valoraciones positivas o negativas en torno a la presencia de niños y niñas migrantes a propósito de las variables de estudio indicadas antes, lo que fluctuaron entre 17 a 39 puntos para la categoría alta percepción; 40 a 61 puntos para la categoría mediana percepción y 62 a 85 para la categoría baja percepción. Finalmente, los resultados de estos instrumentos fueron trabajados con el sistema informático SPSS.

En forma adicional se aplicaron entrevistas en profundidad a 7 docentes de aula de las asignaturas de computación, educación física, matemática, inglés e historia, seleccionados a partir de criterios de conveniencia (Alaminos, 2006), además de los/as Jefes/as de la Unidad de Técnico Pedagógica de ambas escuelas, con el fin de indagar y contrastar los resultados obtenidos en el cuestionario. Las entrevistas fueron sujetas a un proceso de validación de contenido a través del juicio de expertos, en que participaron dos académicos con manejo tanto del tema como de metodología de la investigación. La información proveniente de las entrevistas fue trabajada a partir del análisis de discurso en atención a sus dimensiones de uso de lenguaje, comunicación, creencias y la interacción en situaciones de índole social (Amezcua y Gálvez, 2002), esto en relación a las variables indicadas anteriormente, vale decir, construcción de comunidad y valores inclusivos, las que provienen de la perspectiva teórica utilizada en la investigación.

\section{Resultados}

\subsection{La construcción de una comunidad inclusiva}

Las encuestas aplicadas ponen en evidencia que en ambos establecimientos existe una alta percepción hacia la presencia de los/as niños/as migrantes en lo que refiere a cada una de las variables y categorías de estudio. En términos generales el $97 \%$ de los y las docentes está dentro de un rango alto de valoración y percepción positiva en torno a su presencia y aporte a la escuela, lo que marca una tendencia que resulta auspiciosa en relación a los fines que persigue la inclusión educativa. En efecto, la disposición al trabajo con la diversidad es un punto inicial que puede derivar en acciones concretas. Las evidencias sostienen que las actitudes positivas hacia la diversidad por parte de los y las docentes son lo más relevantes en escuelas que planifican y se proyectan en la lógica inclusiva (Poblete y Galaz, 2015).

Respecto de cada una de las variables de estudio los resultados son similares, dado que se mueven dentro de rangos altos de valoración y percepción positiva, al menos desde lo que arrojan las encuestas aplicadas. En el caso concreto de la construcción de comunidad, y en particular en lo relativo a la participación, una mayoría significativa sostiene que le parece relevante la participación de los/as migrantes en la escuela y que incluso son ellos quienes promueven acciones para que así sea. Al respecto, un 71\% de los y las docentes de ambos centros educativos está de acuerdo y muy de acuerdo con que los y las migrantes tienen la posibilidad de involucrarse en la toma de decisiones de la escuela.

Sin embargo, aquí aparece un punto relevante, dado que las entrevistas en profundidad advierten que esa participación queda restringida a determinados espacios y actividades, en particular aquellas que son propios de las expresiones culturales de cada uno de los países representados, tales como ferias multiculturales, festivales culinarios, etc. 
El año pasado hacíamos disertaciones sobre sus países, sobre el lugar donde ellos nacieron, sus costumbres, sus trajes típicos, sus comidas, y había una muestra, era un trabajo familiar, venían los padres, la mamá o el papá o un familiar cercano, si no podían los papas, y el niño se presentaba a disertar con su traje típico con su mapa y hablaba de los límites, de los lugares más lindos, de las comidas, de todo, es un espacio, fue muy importante y muy lindo ese trabajo. (Profesora de Matemáticas)

Como se desprende de este relato hay una tendencia a hacer visible la diferencia -y hacerla participar- a través de su folclorización, sin incidencia en las decisiones más relevantes que tiene la escuela. Por cierto, esto responde a una lógica de transformación curricular asociada a lo que se conoce como enfoque de las contribuciones (Banks, 1989, 1994; Escarbajal, 2011; Gibson, 1991; Itarte, 2005; Lluch, 2005; McCarthy, 1994; Muñoz, 2001; Poblete, 2009), que aboga por la incorporación de contenidos tales como festividades y celebraciones propias de los grupos étnicos y minoritarios, sin intervenir en profundidad el currículum o la propia estructura escolar. Y aunque resulta positivo dado que se trata de un avance, no genera impactos significativos si no está asociada a una estrategia inclusiva de largo plazo.

Al consultar si se solicita la opinión de los estudiantes migrantes sobre cómo debería mejorar el centro educativo, el $73 \%$ de los/as docentes de ambos establecimientos responde de forma positiva, aunque nuevamente en las entrevistas aparecen matices que sitúan la capacidad de decisión de los y las migrantes en ámbitos acotados que resultan poco relevantes. Por ejemplo, en una de las entrevistas se menciona al centro de estudiantes como instancia participativa, pero no tiene carácter resolutivo, aunque si se reconoce que al menos es la oportunidad de plantear determinados temas a la dirección de las escuelas. Esto puede ser problemático si se considera que la participación escolar no es solo una práctica derivada de un principio de funcionamiento político, sino que debe aportar a la eficacia de los procesos educativos (Jara, 2011).

En la misma lógica el $62 \%$ de los y las docentes está de acuerdo y muy de acuerdo en que se cuenta con una variedad de oportunidades para los y las migrantes de involucrarse en la escuela, sin embargo, la información obtenida en las entrevistas marca un nuevo contrapunto, toda vez que se indica que esos espacios son restringidos y poco relevantes para el desarrollo de ambos centros educativos.

En el marco educativo nacional existen órganos al interior de las escuelas que requieren participación de todos los actores, como por ejemplo los consejos escolares. ${ }^{1}{ }^{\mathrm{Y}}$ aunque se afirma que niños y familias migrantes participan de ellos, también se reconoce que lo hacen de forma restringida, dado que son espacios informativos y no consultivos ni resolutivos:

Los apoderados en ese caso pueden participar directamente en los consejos escolares, en el centro de padres, y bueno, en toma de decisiones no mucho en realidad... son más informativos, pero no consultivos ni de toma de decisiones. (Profesora de Historia)

Los procesos de inclusión requieren que la estructura escolar refleje la diversidad propia de la escuela, a través de la participación activa de todos los grupos en la toma de decisiones de forma tal que se resalta la relevancia de la dimensión comunitaria de la organización (Obando, 2008). Por lo mismo, para el caso de los centros en estudio, resulta clave avanzar hacia formas de participación activa dado que esta es reconocida como una

\footnotetext{
${ }^{1}$ En el marco de la educación chilena los consejos escolares son instancias que tienen por objetivo estimular y canalizar la participación de la comunidad educativa en el proyecto educativo y en las demás áreas que estén dentro de las esferas de sus competencias (Artículo 15, Ley 20.370).
} 
"seña de identidad propia de la educación inclusiva, lo que supone dar voz a todos los miembros de una comunidad educativa para que colaboren en la toma de decisiones que guían la vida de un centro" (Sarto y Venegas, 2009, p. 10).

Respecto de las relaciones al interior de las escuelas entre estudiantes chilenos/as y extranjeros/as, de parte de los y las docentes existe la convicción que estas habitualmente son de colaboración en el marco de los procesos de enseñanza aprendizaje, lo cual es corroborado por la información obtenida en las entrevistas. En este punto, hay una clara concordancia entre la información proveniente de ambas fuentes.

De esta forma, las relaciones al menos desde lo académico se dan desde la cooperación entre estudiantes chilenos/as y extranjeros/as, lo que resulta positivo dado que se reduce la intolerancia, la frustración e incluso pueden derivar en lazos de amistad:

Ellos mismos van generando grupos de amistad... su trabajo colaborativo se da no pensando en que me voy a juntar con el colombiano, sino con este niño que sabe más que yo, pero no por una cuestión de nacionalidad. (Profesor de Matemática)

Este tipo de relaciones de cooperación son un buen punto de inicio para los procesos de adaptación que deben vivir niños y niñas migrantes cuando se insertan en un sistema educacional distinto, lo cual es especialmente útil para quienes no manejan el idioma, como ocurre con niños y niñas haitianas:

\begin{abstract}
Por ejemplo, si tenemos un niño que no entiende el idioma, el haitiano, por ejemplo; hay niñas que se hacen cargo de manera autónoma de esos compañeros. Entonces por ejemplo yo he visto en el comedor o cosas así más fuera del aula, las niñas se sientan y le explican a la compañera con gestos que es lo que están tratando de decirle...entonces ellas mismas tratan, buscan estrategias de adaptar y acoger a esta persona, eso es natural no es guiado por ningún profesor ni nada. (Profesora de Historia)
\end{abstract}

En cuanto a las formas de interacción que se producen en la escuela, los/as docentes describen que las relaciones entre chilenos/as y extranjeros/as se mueven dentro de lo "normal". El 78\% de los y las docentes estuvo muy de acuerdo en que se establecen relaciones de amistad entre ambos grupos de estudiantes, aunque identifican algunas tensiones y roces que cristalizan en el hecho que niños y niñas tienden a agruparse por nacionalidad.

Esto es coherente con los resultados de otras investigaciones realizadas en escuelas que han advertido que por lo general los/as niños/as chilenos/as y extranjeros/as más pequeños/as no tienen problemas en vincularse entre sí, sin embargo, a medida que crecen comienzan a relacionarse casi exclusivamente con sus connacionales (Poblete y Galaz, 2015). Al menos en el caso de este estudio aparece mucho más vinculado al colectivo colombiano:

\footnotetext{
Los colombianos son muy de piel, entonces ellos hacen como un clan, que es distinto a lo que somos los chilenos, los peruanos y los ecuatorianos...pero los colombianos en sí ellos generan un clan, ellos hacen, asi como un grupo, se juntan entre colombianos. (Profesora de Computación)
}

En ambos establecimientos se constató la presencia de sobrenombres o apodos derivados de la nacionalidad. El uso de etiquetas aparece como un factor relevante en el marco de las relaciones entre estudiantes. En forma adicional el color de la piel también determina formas de discriminación:

Bueno acá en Chile en general ya hay mucho clasismo respecto al color de piel, (...) por el color de piel, el color de piel es para mí, es lo que yo he observado, más marca la diferencia o genera exclusión. (Profesora de Educación Física) 
Otras investigaciones coinciden en este punto (Pavez, 2012; Poblete y Galaz, 2015; Tijoux, 2013) y sitúan a niños/as afrodescendientes, y en particular colombianos y haitianos, como blanco de las burlas de sus pares.

De acuerdo a la percepción general de los docentes las relaciones entre estudiantes en estos centros educativos son positivas, situándose por sobre lo que ocurre con la participación y la posibilidad de incidir en las decisiones de la escuela, que aparece como un elemento deficitario. Sin embargo, cabe preguntarse si solo existe la capacidad de identificar las formas de discriminación más evidente, no reconociendo que también operan otras más sutiles que tienden a ser normalizadas en la cotidianeidad de las escuelas (Conde, 2014), e incluso se justifican como algo normal de la edad de niños y niñas (Poblete y Galaz, 2015). Más concretamente, las escuelas tienden a naturalizar ciertos comportamientos y asumirlos como algo propio de las relaciones, sin considerar que se trata de discriminaciones que afectan a quienes las viven.

\subsection{Valores inclusivos}

La presencia de valores en las escuelas es clave para la incorporación de acciones destinadas a la creación de una cultura inclusiva, dado que estos son la base de todo lo que hacen los centros educativos (Andrés y Sarto, 2009). Aunque estos valores son múltiples y van desde la igualdad y el respeto por los derechos hasta la confianza, honestidad y afecto (Booth, 2010), existe uno que implica al resto y es central en este tipo de procesos. Se trata del respeto a la diversidad y que en el caso de esta investigación se vio reflejado en la incorporación en los documentos oficiales de las escuelas que declaran explícitamente trabajar con ella. En efecto, el $100 \%$ de los y las docentes encuestados señala que los proyectos educativos (Proyectos Educativos Institucionales) de sus respectivas escuelas tienen apartados específicos que se comprometen con el desarrollo de acciones concretas tendientes al respeto de la diversidad. Muchas de estas acciones se orientan, tal como se mostró en el apartado anterior, a visibilizar la cultura de los y las migrantes a través de las "ferias" y otro tipo de expresiones de carácter folclórico. Por lo mismo, el 97\% de los y las docentes está de acuerdo con que se considera y valora la cultura de los diferentes colectivos presentes en la escuela, lo que se refleja en el trato que reciben de parte de ellos/as:

\footnotetext{
Aquí son todos exactamente iguales, son todos tratados iguales, todos con el mismo tiempo, con el mismo cariño, con el mismo respeto, con el mismo amor, para mí son todos niños, yo no puedo hacer diferencias con uno o con otro, porque es negro, porque es blanco, porque es rubio, porque es moreno, porque es de ojos azules, nada, son todos iguales. (Jefa de UTP)
}

Ahora bien, el respeto por las diferencias que representan los y las estudiantes migrantes también se asocia a la reducción de toda forma de racismo y discriminación, lo que es corroborado por el $97 \%$ de los y las docentes que indica estar muy de acuerdo y de acuerdo con que se realizan acciones de carácter institucional en esa línea. Sin embargo, esta percepción positiva respecto de lo que hace la institución y los mismos docentes, contrasta con algunos relatos que indican que existen en ambos establecimientos profesores/as que discriminan en el trato a sus estudiantes extranjeros/as. Aunque puede tratarse de casos excepcionales, debe ser parte de la preocupación constante de la dirección de las escuelas y de quienes ejercen posiciones de liderazgo para su erradicación.

En torno a la generación de igualdad de oportunidades, se asume que se logra cuando la escuela trabaja brindando diversas alternativas pedagógicas para garantizar el acceso al 
currículum, el aprendizaje y la participación activa de todos y todas. Sobre este punto aparecen elementos que son relevantes, en especial en cuanto a la percepción que los propios docentes tienen acerca de los contenidos que deben entregar y que a su juicio son "muy elevados", lo que genera una distancia entre estudiante chilenos/as y extranjeros/as.

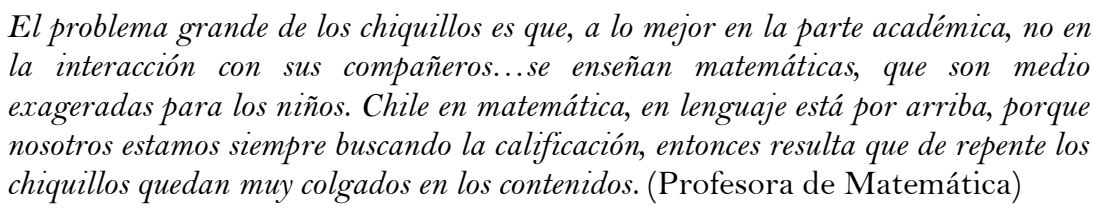

Esta suerte de "déficit" -que es compartido por casi el 50\% de las y los docentes- es explicado por el origen nacional e incluso por el lugar del cual provienen dentro de sus respectivos países.

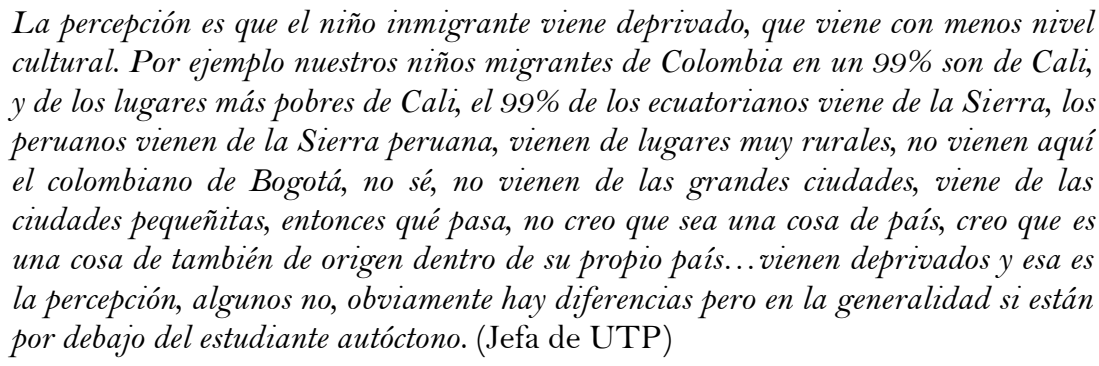

En este relato queda de manifiesto que se reconocen diferencias territoriales y de origen socioeconómico, de forma tal que los estudiantes que provienen de zonas rurales y empobrecidas son quienes pueden presentar mayores dificultades para acceder el currículum y los aprendizajes esperados. Esto pone en evidencia que muchas veces se considera que las culturas minoritarias del alumnado de origen inmigrante se encuentran en desventaja o déficit frente a la cultural mayoritaria, lo que por cierto puede influir en las expectativas que los y las docentes tienen hacia sus estudiantes (Carrasco, 1998; Jordán, 1994; Ogbu, 1991; Poblete, 2011 Salas et al., 2012). En ese marco, también se identifican culturas que presentan mayores dificultades para insertase en la lógica de las normas de conducta, como es el caso de venezolanos/as y dominicanos/as:

Ellos se ríen, son súper alegres, pero hay un tema con ellos que hay que afianzar normas, reglas, sistematizar hábitos...no sé, es un poquito más complejo. (Profesora de Inglés)

Sin embargo, también es preciso consignar que esas culturas deficitarias en lo disciplinario también tienen otras aptitudes, dado que a quienes provienen de Venezuela se les atribuye una mejor preparación académica que el resto de los y las migrantes.

Lo anterior plantea la pregunta si los contenidos establecidos en la educación chilena tienen un estándar más elevado en comparación a los países vecinos, y si este aspecto influye en que los docentes tengan la percepción que los/as estudiantes migrantes llegan con un rendimiento o recorrido académico inferior que los sitúa con un déficit frente a sus pares. Esta reflexión adquiere importancia al observar que fue un discurso recurrente en los y las docentes de ambas escuelas.

En esa lógica, también se indagó en la entrega de apoyos a los y las estudiantes extranjeros para favorecer su proceso de aprendizaje. El 100\% de los y las docentes está de acuerdo y muy de acuerdo en que la escuela da el mismo apoyo a todos los estudiantes, independiente de sus diferencias culturales. Sin embargo, desde las evidencias obtenidas en las 
entrevistas aparece que se relaciona la entrega de apoyos con los proyectos de integración escolar, las duplas psicosociales y los talleres de reforzamiento.

Todos los apoyos que nosotros entregamos son a base de la problemática que nos van presentando a diario, y que no son problemáticas para nosotros, son desafíos. Hay PIE (Programas de Integración Escolar), hay especialistas en el tema de dificultad de aprendizaje, dificultad del habla, ellas apoyan a la profesora en el aula y con las profesoras de primer ciclo y de segundo ciclo hacen un seguimiento, pero si hay apoyo también hay apoyo de psicóloga y asistente social, o sea nosotros contamos con la suerte de tener un gran recurso humano en el PIE y el apoyo psicopedagógico. (Profesora de Computación)

Una de las características de estos programas es la agrupación de estudiantes de acuerdo a criterios de origen o rendimiento académico, siendo separados del grupo mayoritario para que reciban apoyos específicos y subsanen las dificultades a nivel pedagógico y de adaptación al entorno escolar.

En esta lógica las dificultades están enfocadas en el estudiante, no en las barreras presentes tanto en la cultura escolar como en el currículo. Otro aspecto a tener en cuenta es que "realizar la separación de los estudiantes pertenecientes a los programas compensatorios interfiere directamente en las posibilidades de interacción e intercambio entre los estudiantes migrantes y los chilenos, limitando las oportunidades de conocimiento y respeto hacia la diversidad cultural” (Jiménez, 2014, p. 417).

De ahí que es preciso generar apoyos no centrados y fijados solo en el alumno y en sus dificultades de aprendizaje, por el contrario, se requiere la modificación de la organización del centro educativo y del aula, así como de la metodología de intervención para asegurar la participación de todos en la dinámica común del aula (Muntaner, 2000).

Finalmente, un punto que aparece como relevante en el proceso de garantizar igualdad de oportunidades, es lo referido a la lengua que se constituye en una barrera, en especial para niños y niñas haitianas, dado que los y las docentes no se encuentran preparados para abordar las necesidades que surgen de esta situación. En este caso es claro que las escuelas requieren apoyos que trascienden su marco de acción y remiten al rol de los municipios o el propio Ministerio de Educación.

\section{Comentarios de cierre}

A partir de los resultados alcanzados en la investigación queda de manifiesto que los y las docentes poseen imaginarios que tienden a atribuir a los y las migrantes una serie de déficits académicos y de comportamiento que dificultan su proceso de inclusión y su plena participación en las escuelas.

Es el caso de las etiquetas que existen en relación a los diferentes colectivos, lo cual ha sido "normalizado" por parte de los y las docentes al asumir que por ejemplo los/as colombianos/as son más "alegres", o los/as venezolanos/as tienen mayores dificultades para comportarse de acuerdo a las normas establecidas.

Todo esto cristaliza en una concepción de la diversidad que representan los y las migrantes como una suerte de obstáculo que debe ser superado. Y la forma de hacerlo es a través de estructuras asimilacionistas que emanan desde la propia idea educativa del país y las escuelas. En efecto, como se indicó antes, existe suficiente evidencia en torno al hecho 
que la escuela en Chile se ordena desde una perspectiva normalizadora y excluyente que deriva en una concepción uniforme de los procesos educativos (Poblete y Galaz, 2007).

Por lo mismo las acciones que toman las escuelas para visibilizar la diversidad se reducen a muestras culinarias o de bailes típicos que terminan folclorizándola, dificultando su uso como herramienta para la transformación curricular y valórica que permita superar las visiones restringidas en torno a ella.

$\mathrm{Al}$ respecto, fortalecer los procesos de formación inicial docente y la formación en ejercicio, en especial en la perspectiva intercultural, es una estrategia que puede ser útil en el contexto actual. Esto no solo para desarrollar actitudes positivas hacia la diversidad, sino en especial para la mejora de las competencias de los y las docentes para llevar a cabo adaptaciones curriculares que permitan visibilizar la cultura de los y las migrantes, no solo como una iniciativa "folclórica", sino como un recurso para el aprendizaje.

Por cierto, esto supone el apoyo de las estructuras políticas, tanto a nivel del Ministerio de Educación y de los Municipios, y sociales, vale decir, universidades y organizaciones de la sociedad civil que pueden favorecer, a través de la generación de redes de apoyo a la escuela, los procesos de inclusión. Lo que no puedo ocurrir, es que los centros educativos se sientan solas en este esfuerzo, como lo han señalado otros estudios realizados al respecto (Poblete y Galaz, 2015).

Aunque queda mucho por avanzar, no se puede desconocer que existe un esfuerzo sistemático por promover, aún en un marco restringido, el respeto a la diversidad y la valoración de las diferencias, evitando el racismo y otras actitudes perniciosas en el marco de los procesos de inclusión educativa. Si bien el límite entre lo correcto y aquello que no lo es tiende a ser difuso y muchas veces se naturalizan ciertas conductas como propias de la edad de niños y niñas, es importante reconocer el valor que los y las docentes le otorgan al respeto y la solidaridad al interior de la escuela como una condición central para un clima adecuado para el aprendizaje. Ciertamente, esas son las bases para aportar a una cultura inclusiva que nos permita dejar atrás como sociedad el racismo, la xenofobia y otras formas de interacción humana que resultan perjudiciales para la convivencia democrática.

\section{Referencias}

Alaminos, A. (2006). El muestreo en la investigación social. En A. Alaminos, y J. L. Castejón (Ed.), Elaboración, análisis e interpretación de encuestas, cuestionarios y escalas de opinión (pp. 41-67). Alicante: Marfil.

Amezcua, M. y Gálvez, A. (2002). Los modos de análisis en investigación cualitativa en salud: Perspectiva crítica y reflexiones en voz alta. Revista Española de Salud Pública, 76(5), 423436. https://doi.org/10.1590/S1 135-57272002000500005

Andrés, M. y Sarto, M. (2009). Escuela inclusiva: Valores, acogida y convivencia. En M. P. Sarto y M. E. Venegas (Coords.), Aspectos clave de la educación inclusiva (pp. 85-118). Salamanca: Instituto Universitario de Integración en la Comunidad.

Banks, J. (1989). Integrating the curriculum with ethnic content: Approaches and guidelines. En J. Banks y C. Mcgee Banks (Coord.), Multicultural education. Issues and perspectives (pp. 189207). Boston, MA: Allyn and Bacon.

Banks, J. (1994). An introduction to multicultural education. Boston, MA: Allyn and Bacon. 
Blanco, R. (1999). Proyecto principal de educación para América Latina y el Caribe. Boletín, 48, 5572.

Blanco, R. (2006). La equidad y la inclusión social: Uno de los desafíos de la educación y la escuela hoy. REICE. Revista Iberoamericana sobre Calidad, Eficacia y Cambio en Educación, 4(3), 1-15.

Booth, T. y Ainscow, M. (2000). Guía para la evaluación y mejora de la ecuación inclusiva. Index for inclusion. Madrid: Consorcio Universitario para la Educación Inclusiva.

Booth, T. (2010). La inclusión como marco para el desarrollo educativo: Transformar los valores en acción. En Fundación PAR (Ed.), Escuelas inclusivas un camino para construir entre todos (pp. 38-58). Buenos Aires: Fundación PAR.

Carrasco, S. (1998). Interculturalitat $i$ educacio. Aportacions per un debat entre l'antropología social y la pedagogía. Barcelona: Universitat Autónoma de Barcelona.

Castro, C. (2012). Inmigración, hoy: El reflejo de las representaciones sociales de los/las docentes en la cultura, la política y las prácticas de la escuela en el marco de la diversidad cultural (Tesis de Magister). Santiago de Chile: Universidad Central.

Campo, M., Álvarez, M., Castro, P. y Álvarez, E. (2005). La situación de los alumnos extranjeros vista por los maestros de primaria. Aula Abierta, 86, 205-218.

Cerrutti, M. y Binstock, G. (2012). Los estudiantes inmigrantes en la escuela secundaria. Integración y desafíos. Buenos Aires: Argentina.

Conde, F. (2014). Desigualdad, discriminación y pedagogía de la igualdad. Actualidades Investigativas en Educación, 14(1), 1-20.

Dyson, A., Howes, A. y Roberts, B. (2002). Una revisión sistemática de la efectividad de las acciones a nivel de la escuela para promover la participación de todos los estudiantes. Londres: Institute of Education.

Echeita, G. (2008). Inclusión y exclusión educativa. Voz y quebranto. REICE. Revista Iberoamericana sobre Calidad, Eficacia y Cambio en Educación, 6(2), 9-18.

Escarbajal, A. (2011). Hacia la educación intercultural. Revista Interuniversitaria, 18, 131-149. https://doi.org/10.7179/PSRI_2011.18.10

Gibson, M. (1991). Minorities and schooling: Some implications. En M. Gibson y J. Ogbu (Coords.), Minorities status and schooling: A comparative study of immigrant and involuntary minorities (pp. 357-381). Nueva York, NY: Garland Publishing,

Hernández, R., Fernández, C. y Baptista, M. (2010) Metodología de la investigación. Ciudad de México: McGraw Hill.

Itarte, R. (2005). Pluralidad y educación. La intercultura como modelo educativo. En T. Fernández y J. García (Coord.), Multiculturalidad y educación: teorías, ámbitos y prácticas (pp. 66-95). Madrid: Alianza.

Jara, C. (2011). Sobre el falso concepto de participación en educación. CISMA. Revista del centro Telúrico de Investigaciones Teóricas, 1, 1-10.

Jiménez, F. (2014). Modelos de gestión de la diversidad cultural para la escolarización del alumnado inmigrante en las escuelas chilenas: Desafíos para la interculturalidad actual. Estudios Pedagógicos, 4O(2), 409-426. https://doi.org/10.4067/S0718-07052014000300024

Jiménez, F. y Fardella, C. (2015). Diversidad y rol de la escuela: Discursos del profesorado en contextos educativos multiculturales en clave migratoria. Revista Mexicana de Investigación Educativa, 20(65), 419-441. 
Johnson, D. (2015). Formar ciudadanos interculturales en un mundo global: Algunas notas desde los estudios curriculares. Revista Diálogo Andino, 47, 7-14. https://doi.org/10.4067/So7 1926812015000200002

Jordán, J. (1994). La escuela multicultural: Un reto para el profesorado. Barcelona. Paidós.

Kaluf, M. (2009). Niños inmigrantes peruanos en la escuela chilena (Tesis de Magister). Santiago de Chile: Universidad de Chile.

Lasso, M. (2015). Cultura inclusiva en la escuela. Revista para el Aula, 14, 24-25.

León, B., Mira, A. y Gómez T. (2007). Evaluación de las opiniones sobre la inmigración y la multiculturalidad en la escuela de alumnos de magisterio. Revista Electrónica de Investigación Psicoeducativa, 5(12), 259-282.

Lluch, X. (2005). Educación intercultural y currículum: Una oportunidad de repensar la cultura escolar. En T. Fernández y J. García (Coords.), Multiculturalidad y educación: Teorías, ámbitos yprácticas (pp. 179-216). Madrid: Alianza.

McCarthy, C. (1994). Racismo y currículo: La desigualdad social y las teorías y políticas de las diferencias en la investigación contemporánea sobre la enseñanza. Madrid: Morata.

Muñoz, A. (2001). Enfoques y modelos de educación multicultural e intercultural. Recuperado de http://www.comadrid.es/webdgpe/Interculturalidad/enfoques.doc

Ministerio de Educación de Chile. (2005). Ordinario $N^{\circ} 07 / 1008$ (1531). Santiago de Chile: MINEDUC.

Ministerio de Educación de Chile. (2016). Ordinario Nº2/000894. Santiago de Chile: MINEDUC.

Ministerio de Educación de Chile. (2017). Recuperado de https://migrantes.mineduc.cl/

Muntaner, J. (2000). Igualdad de oportunidades en la escuela de la diversidad. Profesorado. Revista de Currículum y Formación de Profesorado, 4(1), 1-19.

Obando, G. (2008). La participación docente en la toma de decisiones desde la visión micropolitica. Revista de Educación, 17(32), 87-108.

Ogbu, J. (1991) Immigrant and involuntary minorities in comparative perspective. En M. Gibson y J. Ogbu (Coords.), Minority status and schooling. A comparative study of immigrant and involuntary minorities (pp. 3-33). Nueva York, NY: Garland Publishing

Ortiz, M. (2008). Inmigración en las aulas: Percepciones prejuiciosas de los docentes. Revista de Sociología Papers, 87, 253-268. https://doi.org/10.5565/rev/papers/v87no.805

Pavez, I. (2012). Inmigración en Chile. Experiencias de la niñez peruana en Santiago de Chile. Revista de Estudios Tranfronterizos, 21(1), 75-99.

Pereira, Z. (2011). Los diseños de método mixto en la investigación en educación: Una experiencia concreta. Revista Electrónica Educare, 15(1), 15-29.

Poblete, R. y Galaz, C. (2007, septiembre). La identidad en la encrucijada, migración peruana y educación en el Chile de hoy. Ponencia presentada en el II Congreso internacional de etnografía y educación, migraciones y ciudadanía. Universidad Autónoma de Barcelona.

Poblete, R. (2011). Género y educación: Trayectorias de vida para ellos y ellas. Revista Latinoamericana de Inclusión Educativa, 5(1), 63-77.

Poblete, R, Galaz, C. y Frías, C. (2015). Inmigrantes en Chile: Oferta programática, participación, inclusión y vulnerabilidad. Investigación realizada para el Ministerio de Desarrollo Social. Documento no publicado. 
Salas, A. y Urbano, C. (2012). La educación intercultural. Percepciones y actitudes del profesorado. Revista Iberoamericana de Educación, 58(1), 1-15.

Sarto, M. P. y Venegas, M. E. (2009). Aspectos clave de la educación inclusiva. Salamanca: Instituto Universitario de la Integración en la Comunidad.

Tijoux, M. (2013). Las escuelas de la inmigración en la ciudad de Santiago: Elementos para una educación contra el racismo. Polis Revista Latinoamericana, 12(35), 287-307. https://doi.org/10.4067/So718-65682013000200013

UNESCO. (2001). Declaración universal de la UNESCO sobre la diversidad cultural. París: UNESCO.

UNICEF. (2004). Convivencia y discriminación en el ámbito escolar. Santiago de Chile: UNICEF.

UNESCO. (2005). Guidelines for inclusion: Ensuring access to education for all. París: UNESCO.

UNICEF. (2011). Convivencia y discriminación en el ámbito escolar. Santiago de Chile: UNICEF.

\section{Breve CV de los autores}

\section{Leydi Patricia Cerón Campo}

Fonoaudióloga titulada de la Universidad del Cauca - Colombia, Magíster en Educación Inclusiva Universidad Central - Santiago de Chile, Diplomada en Trastorno Específico del lenguaje de la Universidad de Chile. Participante del grupo de gestión de la sociedad educacional Carlos Salinas; Responsable de diagnóstico y tratamiento de niños con alteraciones de lenguaje y asesoría a docentes en el manejo de trastornos de lenguaje en el aula. ORCID ID: 0000-0002-2243-6310. Email: leydiceron@gmail.com

\section{Marly Pérez Alvarado}

Fonoaudióloga titulada de la Universidad del Cauca - Colombia, Magíster en Educación Inclusiva, Diplomada en Trastorno Específico del lenguaje de la Universidad de Chile; se desempeña como profesional en programa de integración escolar en establecimientos de educación Pre-básica y Básica. Encargada de la coordinación de profesionales en la intervención de estudiantes con necesidades educativas especiales derivadas de Trastorno del Espectro Autista y Discapacidad Intelectual en la Corporación Educacional Laudelina Araneda, Co-terapeuta en el Programa de Equinoterapia, Centro EQQUS. ORCID ID: o000-000 1-7041-8689. Email: marlyperez85@gmail.com

\section{Rolando Poblete Melis}

Doctor en Antropología Social y Cultural y Master en Investigación de la Universidad Autónoma de Barcelona, Licenciado en Filosofía por la Universidad Católica de Valparaíso. Es académico de la Facultad de Ciencias de la Educación de la Universidad Central de Chile e investigador del Centro de Desarrollo e Innovación en Educación Inclusiva de la misma universidad. Integrante del núcleo interuniversitario Estudios Críticos de la Diversidad, sus áreas de trabajo son políticas públicas, migraciones, diversidad, género y educación. Sus últimas investigaciones y publicaciones están asociadas a los procesos de inclusión de niños y niñas migrantes en escuelas y en especial al trabajo con la diversidad que llevan a cabo los centros educativos. ORCID ID: 0000o002-7799-7190. Email: rpobletem@ucentral.cl 